\title{
MOBILISATION DANS LES CAMPAGNES TOULOUSAINES : CONFLIT D'AMENAGEMENT ET TENSIONS LOCALES
}

\author{
Pierre PISTRE \\ Université Paris Diderot (Paris 7) \\ UMR8504 - Géographie-cités \\ pierre.pistre@parisgeo.cnrs.fr
}

\section{Résumé :}

Au cours des années 2000, les campagnes toulousaines ont été le théâtre d'une mobilisation d'envergure contre un projet d'aéroport. Cet évènement est ici utilisé comme un prisme d'observation pour analyser les transformations démographiques et sociales en cours dans ces espaces ruraux mobilisés. Une attention particulière est accordée aux profils des opposants, collectifs et individuels, élus et associatifs. Si ce mouvement a initié des proximités et des formes de sociabilités originales, il révèle surtout la prégnance de fortes tensions et fractures locales, au sein d'espaces certes en reprise démographique mais où le vivre ensemble reste largement à construire.

Mots-Clés : Conflit d'aménagement, Campagnes, Sociabilités rurales, NIMBY, Toulouse.

\section{$\underline{\text { Abstract: }}$}

This article presents a geographical analysis of a mobilization against a new airport project in Toulouse's countryside, during the 2000's. We mainly focus on key mobilization's actors, individual and collective, associations and elected representatives. This conflict is used to analyse demographic and social transformations in rural areas mobilized. If it reveals news proximities and kinds of sociability, strong local tensions stay really meaningful in which shows still difficulties of shared lives in peripheral countrysides.

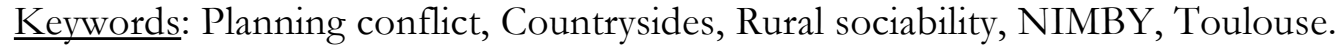

Au cours des années 2000, les campagnes toulousaines ont été le théâtre d'une forte opposition au projet d'un nouvel aéroport. Des rumeurs parcouraient ces territoires depuis plusieurs décennies, alimentées par de nombreuses réflexions aménagistes. En janvier 2003, la préfecture lançait une concertation officielle sur la base de nouvelles études ${ }^{1}$. Les débats se sont cristallisés alors sur l'utilité d'un tel projet et les lieux d'implantation envisagés (Carte 1).

Dans la lignée de précédentes mobilisations régionales (camp militaire du Larzac, centrale nucléaire de Golfech, tunnel du Somport), un important mouvement de contestation se mit en place. Des collectifs associatifs et d'élus ont été créés pour coordonner les revendications et les actions (réunions, pétitions, manifestations). Les rues de Toulouse le 15 mars 2003, et de Montauban le 17 mai 2003, ont rassemblé notamment plus de 15000 personnes venues des campagnes voisines pour protester contre ce projet d'aéroport.

\footnotetext{
${ }^{1}$ Cf. ADPi. 2001. Synthèse : Nouvelle plate-forme aéroportuaire en région toulousaine, 25 p ; DRE Midi-Pyrénées. 2003. Avenir de la plate-forme aérienne régionale de Toulouse, $43 \mathrm{p}$.
} 
Carte 1. Sites pressentis pour accueillir un nouvel aéroport dans la région toulousaine (juin 2001)

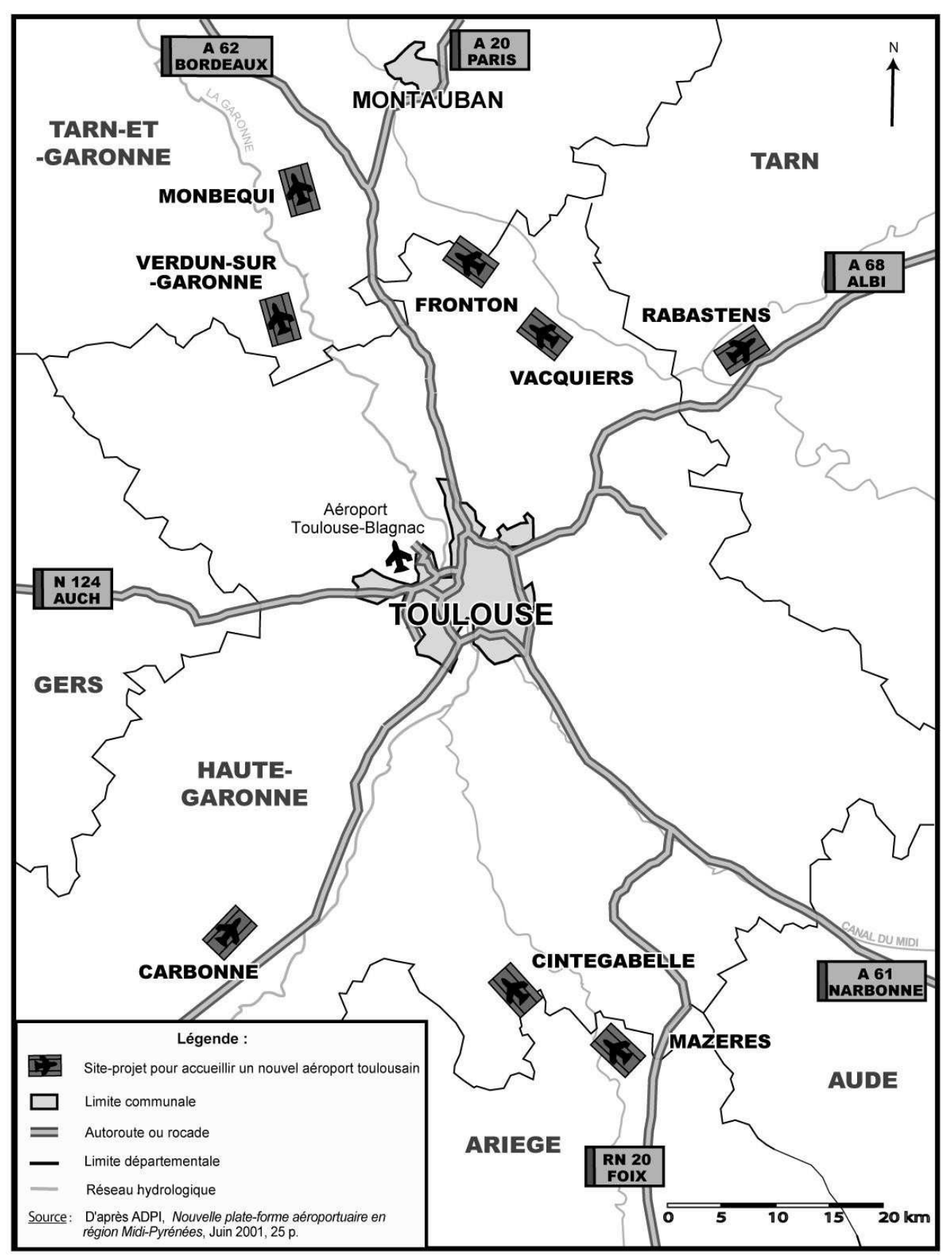

Cet article s'intéresse aux bouleversements démographiques et sociaux en cours dans les campagnes toulousaines (périphérie lointaine : 25-50 km). Ces régions historiquement agricoles connaissent, depuis les années 1980-1990, une certaine renaissance et une progression de leur fonction résidentielle (Kayser, 1990 ; Perrier-Cornet et al., 2002). Des populations, aux expériences de vie majoritairement citadines, viennent s'y installer. Elles recherchent un cadre de vie campagnard, une relative proximité avec Toulouse et des biens immobiliers plus abordables. Ces nouveaux arrivants participent pleinement à une recomposition des sociétés locales, marquées notamment aujourd'hui par des clivages entre populations d'origines, d'âges et de profils socioéconomiques hétérogènes (Arlaud et al., 2005 ; Bossuet, 2007). De nombreuses tensions locales modifient en effet les sociabilités rurales contemporaines, au sein d'espaces qui connaissent une reprise démographique effective, toujours plus loin des villes (Laganier et al., 2009). 
La mobilisation contre l'aéroport a forgé une organisation spatio-temporelle singulière au sein des campagnes toulousaines. Trois périodes successives ont marqué son déroulement : une phase initiale de jeunesse (rumeurs, premières semaines) faite de réactions vives et spontanées ; un deuxième temps de maturation (structuration du mouvement, création de collectifs régionaux ${ }^{2}$, réalisation de contre-expertises ${ }^{3}$ ), qui correspond à une montée en généralité de l'argumentaire des opposants (Lolive, 1997) ; enfin, une période de mise en veille, depuis 2005-2006, qui coïncide avec une suspension progressive du projet. Cette mobilisation s'est appuyée sur une forte inscription locale, par la création de dizaines d'associations dites citoyennes et par le vote de motions d'opposition au sein des conseils municipaux. La contestation a été très prégnante au nord de l'agglomération où un important maillage spatial s'est rapidement structuré (Carte 2).

Certes étendue, la géographie de la contestation n'en a pas moins été inégale. Elle s'est principalement cristallisée autour et à proximité des 8 sites pressentis (Lecourt et al., 2004). L'aménagement en question, sa forme et la linéarité des couloirs aériens induits ont plus précisément concentré les mécontentements de manière elliptique autour des sites-projets. De plus, les représentations associées à ce moyen de transport (bruit, pollution, odeur) et les gênes envisagées ont amplifié cette structuration du mouvement.

\section{Carte 2. Géographie de la mobilisation au nord de l'agglomération toulousaine (2003)}

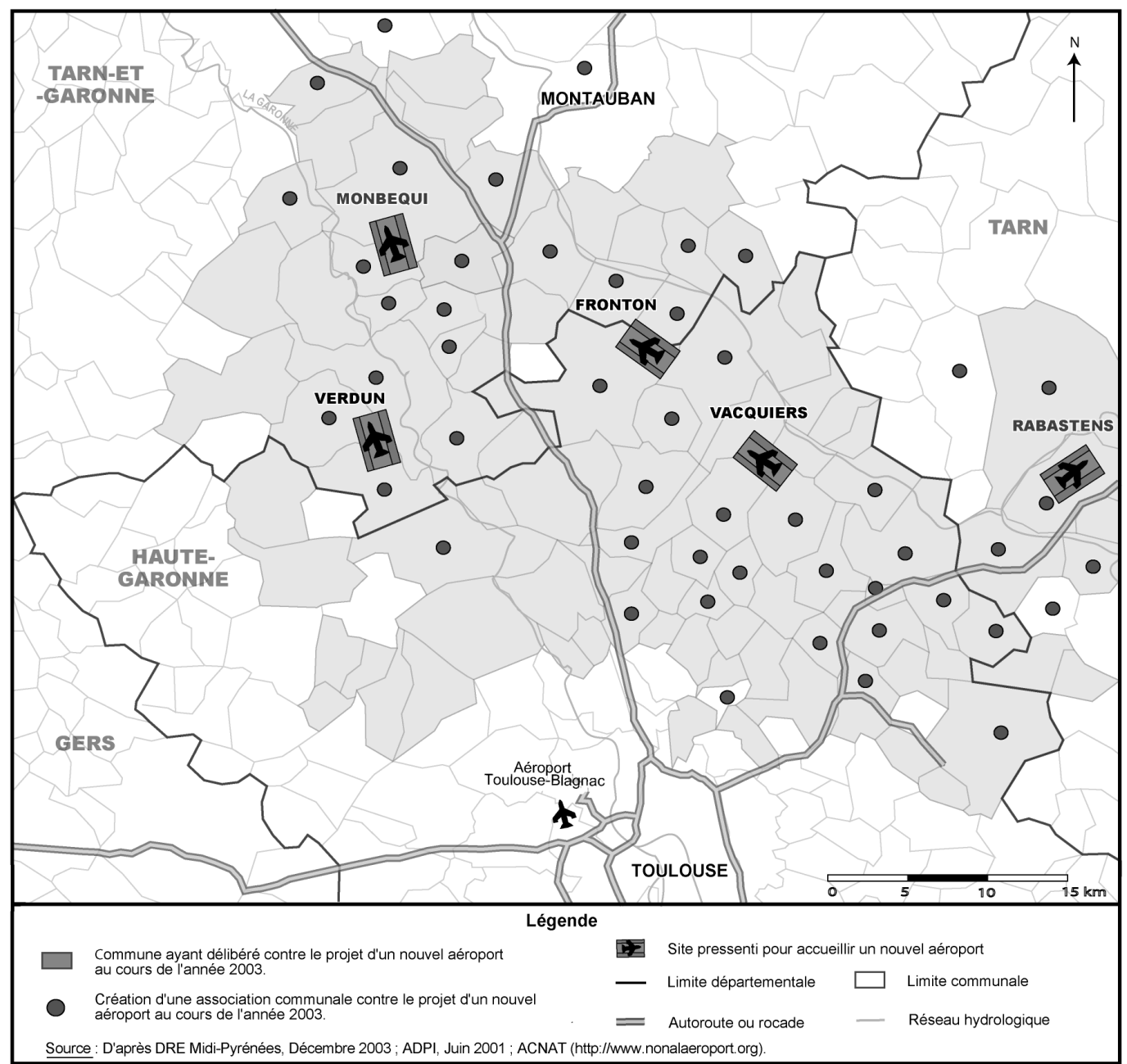

\footnotetext{
${ }^{2}$ Principalement le C-pannet (Contre le Projet d'Aéroport dans le Nord et le Nord-Est Toulousain), le 23 Janvier 2003, et l'ACNAT (Collectif des Associations Contre un Nouvel Aéroport Toulousain), le 6 Février 2003.

${ }^{3}$ Cf. ACNAT/C-PANNET. 2003. Nouvel aéroport toulousain? Contribution citoyenne (Le tout aérien : Non; le bon sens citoyen : Oui), 88 p ; ITA. 2003. Suivi du dossier: Avenir de la plate-forme aérienne régionale, $30 \mathrm{p}$.
} 
L'officialisation du projet, le 13 janvier 2003, marque le point de départ d'une diffusion rapide de la mobilisation, selon des processus d'extension hiérarchique et de contagion, de proche en proche, à partir de sa géographie polycentrique (Saint-Julien, 1985 ; Lecourt, 2003) (Carte 3). Les communes autour de Fronton ont lancé le mouvement. Une revendication centrale concentrait alors leurs préoccupations: la défense de leur vignoble AOC. Ce premier foyer d'opposition a aussi été celui de fortes personnalités, associatives et élus, qui sont devenues les symboles régionaux de la mobilisation. L'organisation de dizaines de réunions publiques, et une médiatisation grandissante, a ensuite fait émerger de nouveaux foyers de contestation autour des autres sites pressentis, notamment au sein des communes rurales les plus peuplées où le mouvement a pu s'appuyer sur des réseaux sociaux préexistants. Ces principaux pôles ont à leur tour mobilisé les communes voisines, et ainsi créer un important maillage d'opposition qui a révélé tout de même des réactivités locales très différentes au cours de l'année 2003.
Carte 3 - Diffusion de la contestation au nord-ouest de Toulouse (2003)

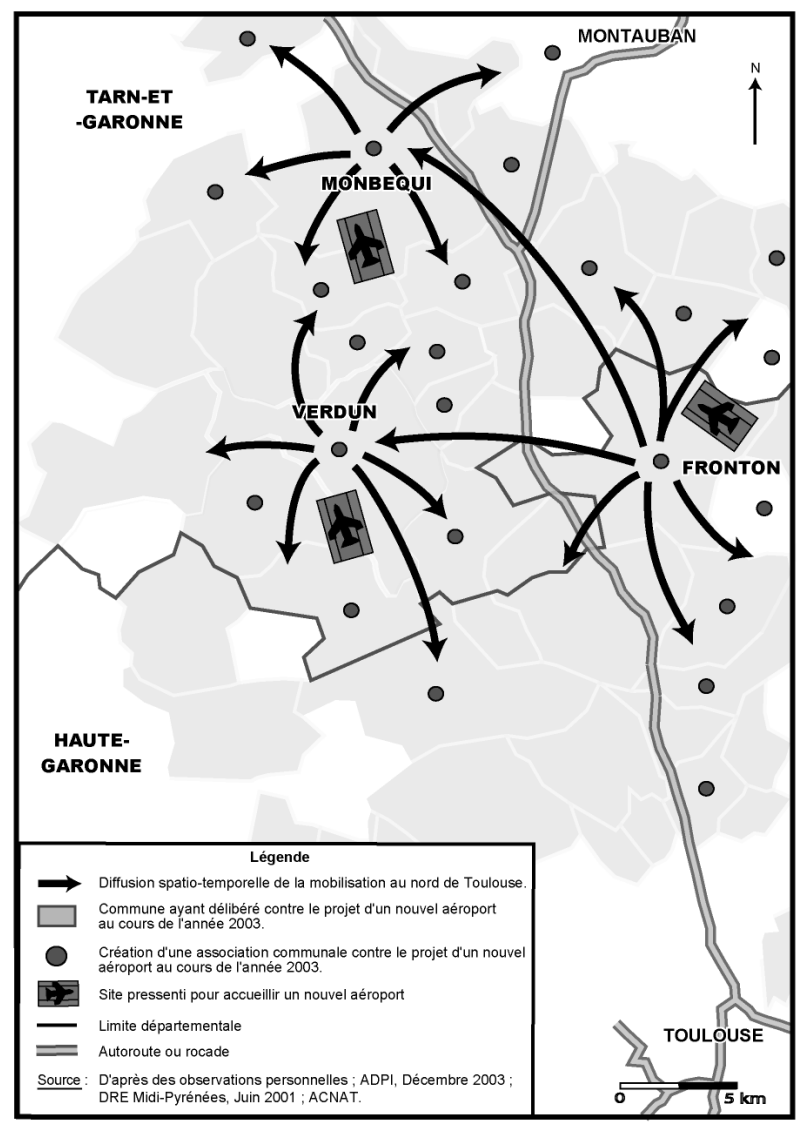

Les acteurs centraux de la mobilisation, individuels et collectifs, associatifs et élus, ont été les principaux instigateurs de sa diffusion spatiale. Suivant une volonté de dissémination la plus large possible, leur objectif a été l'information massive des populations concernées. De nombreux moyens d'action ont été utilisés à cet effet (réunions, présences sur les marchés, tracts dans les commerces), et notamment plusieurs formes d'affichage (Document 4). Des autocollants ont été apposés sur les voitures et les boîtes aux lettres, des banderoles accrochées aux poteaux électriques et disposées le long des axes de circulation, aux entrées des villages. Tels des marqueurs territoriaux de la désapprobation locale, ces outils de communication ont contribué à une large sensibilisation des habitants et des populations de passage.

\section{Document 4-Affichages de l'opposition locale au projet d'aéroport}
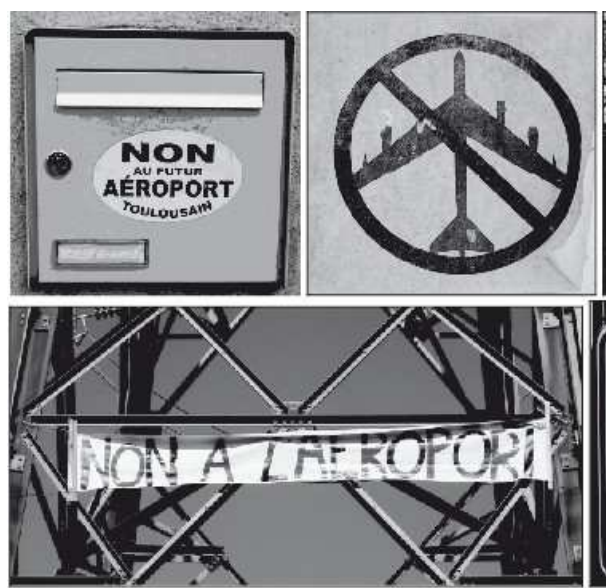

4

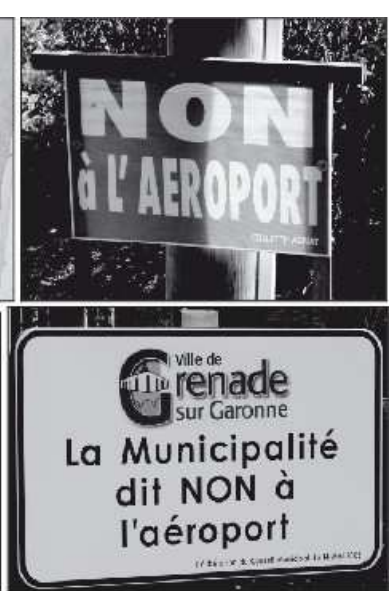

La Municipalité dit NON à I'aéroport 
Les études rurales contemporaines, sauf exceptions notables (Bossuet, 2007 ; Simard, 2007 ; Torre, 2008), abordent peu l'analyse des conflits sociaux et des conflictualités locales. Des recherches sur les aéroports, leurs nuisances et les conflits qu'ils engendrent, ont démontré leurs forts impacts territoriaux, psychologiques et encore politiques (Martinez, 2001 ; Faburel, 2003a ; Faburel, 2003b ; Leroux, 2003 ; Halpern, 2007), mais peu ont étudié les incidences spécifiques des phases d'élaboration et de contestation de ces équipements dans des contextes de rurbanisation.

Notre objectif sera d'analyser comment la mobilisation contre le projet d'un nouvel aéroport révèle des recompositions démographiques, sociales et culturelles importantes au sein des campagnes toulousaines. Quels rôles ont notamment joué les nouveaux arrivants ? Dans quelle mesure leurs origines et leurs expériences citadines ont affecté le déroulement de la mobilisation ? Nous analyserons également les capacités innovatrices de ce mouvement, sa propension à fédérer des populations hétérogènes (âges, origines, statuts sociaux) (Melé, 2003). Notre démarche s'inscrit dans une approche constructive des conflits, afin de récuser une seule lecture " nimby-iste ${ }^{4}$ des conflits d'aménagement, vision simplificatrice et stigmatisante (Simmel, 1995 ; Jobert, 1998). Ils font partie intégrante des nouveaux mouvements sociaux qui ont émergé dans les années 1970, autour d'enjeux environnementaux et de sociétés (Touraine, 1982). Ils peuvent certes révéler des attitudes protectionnistes mais ils ne doivent être réduits à ces seuls positionnements.

Les analyses suivantes sont principalement tirées d'enquêtes menées à proximité directe d'un site pressenti, au sein du canton de Verdun-sur-Garonne (nord-ouest de l'agglomération toulousaine, département du Tarn-et-Garonne) (Pistre, 2006). Des entretiens semi-directifs, auprès de 18 individus ou couples ( $c f$. Annexe 1), et la participation à plusieurs réunions, débats et manifestations, ont permis une étude de la mobilisation dans son environnement local. Nous nous appuyons également, de manière préalable, sur un historique du projet d'aéroport et de l'opposition qu'il a suscitée, réalisé à partir d'une recherche documentaire approfondie (sites internet, rapports, extraits de journaux, publications municipales).

\section{PROFILS DES OPPOSANTS : QUI S'INVESTIT ET POUR QUELLES RAISONS ?}

Ce mouvement s'est construit autour de deux principaux piliers : d'un côté les élus, constituant un véritable lobbying politique, et de l'autre, les associations dites citoyennes, plutôt chargées de son fonctionnement quotidien et de l'organisation des actions. Si cette structuration bicéphale s'est avérée complémentaire et assez efficace à l'échelle régionale, les études de cas font apparaître des ententes locales très différentes. Au sein des principaux pôles ruraux, leurs relations ont souvent été distantes voire conflictuelles, révélant des visions divergentes de la mobilisation, mais aussi des tensions autour de transformations plus profondes. En étudiant ce mouvement d'opposition dans son environnement local, son inscription dans la vie sociale et politique des espaces concernés, cet évènement ponctuel devient un bon analyseur des changements en cours.

Dans un premier temps, l'identification de profils d'opposants, surtout parmi les plus actifs, renseigne sur les forces en présence, leurs origines, leurs trajectoires de vie et leurs places dans le déroulement du mouvement. La recherche de récurrences sociologiques permet notamment d'éclairer des degrés individuels de mobilisation très hétérogènes ( $c f$. Annexe 1 ). Même si un consensus large de refus du projet s'est exprimé localement, une part importante de la population, difficilement quantifiable, se distingue par sa faible implication. D'autres se sont au contraire beaucoup engagés (actifs/responsables des structures locales, régionales), alors que certains ont adopté des attitudes plus intermédiaires, de soutien du mouvement, mais participant seulement aux principales actions. Les analyses qui suivent ne peuvent néanmoins négliger totalement le rôle des sentiments, des ressentis de chacun, pour expliquer ces degrés d'engagement :

\footnotetext{
${ }^{4}$ L'acronyme NIMBY signifie Not In My Back Yard, soit « Pas dans mon jardin » en français.
} 
"Quand je suis allé à la première réunion, je suis tombé des nues [...] Je me suis dit : c'est pas possible qu'on fasse un truc comme ça, [...] Tu dois t'engager. Ce projet est lamentable. Il faut prendre tes responsabilités. C'est comme ça que j'ai commencé. Pourquoi à ce moment là et pas pour d'autres sujets? Je ne sais pas, peut être l'énormité de la chose! » (Entretien n¹6).

\section{Proximités générationnelles et sociales des opposants}

L'âge apparaît comme un premier indicateur discriminant qui révèle des effets générationnels indéniables. Les 30-40 ans ont été fortement mobilisés au sein des associations locales. En couple, avec de jeunes enfants, souvent installés de manière récente, ils ont été particulièrement dynamiques au début du mouvement. A l'origine de la création de plusieurs structures locales, réactifs et accrocheurs, ils ont apporté de la vitalité à la mobilisation, même si certains ont pu s'essouffler et la délaisser avec son prolongement :

"Si ily avait eu moins de jeunes, la mobilisation aurait été moins forte » (Entretien $n^{\circ} 7$ ).

Les jeunes retraités (55-65 ans) constituent un deuxième groupe d'âge clé. Fortement représentés, ils ont souvent géré la mobilisation locale, associative et municipale, en adéquation avec un temps libre récemment dégagé, des envies de nouvelles activités et des compétences à mettre à leurs yeux au service de la collectivité. Anciens instituteurs, enseignants, cadres ou hauts fonctionnaires, les plus actifs ont trouvé dans l'opposition au projet d'aéroport une scène d'engagement propice à leur épanouissement personnel, et une forme de responsabilité publique en adéquation avec leurs aspirations intellectuelles et culturelles (Sauvée, 2004).

Les profils de classes moyennes et supérieures, qui apparaissent à travers le groupe des jeunes retraités, ont plus globalement été surreprésentés dans la mobilisation. Des travailleurs indépendants, des ingénieurs, des employés ou encore des chercheurs se sont également impliqués dans le mouvement. Ils ont en commun une situation économique relativement confortable, malgré des différences de revenus importantes :

"C'est les gens établis, avec une situation stable, qui pewvent penser à s'impliquer là dedans. Pour d'autres, les priorités sont différentes 》(Entretien $n^{\circ} 9$ ).

Propriétaires ou en cours d'acquisitions immobilières, ils entendent défendre les sacrifices, les engagements consentis pour (re)venir s'installer dans les campagnes toulousaines. Le projet d'aéroport remet fortement en cause leur projet de vie, qui repose souvent, en premier lieu, sur la restauration d'anciennes fermes et/ou de maisons familiales.

\section{Des éléments du contexte régional propices à la mobilisation}

Une analyse des profils d'opposants fait également apparaittre la prégnance de particularités régionales. Parmi les plus investis, nous retrouvons tout d'abord des habitués d'autres mouvements sociaux, qui ont été (sont) particulièrement marquants à l'échelle régionale, à commencer par la mobilisation contre la centrale nucléaire de Golfech, dans les années 1970-1980. Plusieurs des retraités gestionnaires, ayant pleinement participé ou qui aiment se remémorer la force de ce mouvement, sa résonance et son intensité, n’hésitent pas à faire une filiation directe avec l'opposition contre l'aéroport. Ce conflit constitue pour eux un point de repère historique majeur :

"Pour la manifestation [Toulouse, le 15 Mars 2003], il y avait 20000 manifestants. Même contre Golfech, y avait pas autant de monde. C'est du jamais vu! »(Entretien n¹3). 
De manière plus contemporaine, certains actifs participent à d'autres mobilisations, par exemple contre les OGM - les actions des «Faucheurs volontaires » sont notamment développées au nord de l'agglomération toulousaine. Plusieurs sont ainsi impliqués dans des pratiques militantes et politiques variées, par exemple écologistes et altermondialistes. Ils sont nombreux à s'être rapidement investis contre l'aéroport, considérant ce projet comme révélateur d'une centralisation à outrance de l'élaboration des grands aménagements publics, et portant atteinte à leur environnement de vie. Ils ont rapidement pris des responsabilités dans les associations et ont souhaité mettre au service de la mobilisation, des expériences, des compétences acquises dans d'autres combats sociaux. Leurs poids effectif et symbolique dans le déroulement du mouvement sont indéniables, mais aussi ambigus. Ils ont par exemple rebuté beaucoup de populations locales moins activistes. Certaines structures associatives ont aussi souhaité se démarquer de ces profils d'opposants :

"L'association de $X$ a été créée la première dans la région, mais là avec des pro-écolos, notamment des Amis de la Terre, avec un vocabulaire etc. J'ai admiré ces gens [...] Au point de vue management, organisation des manifs, ils savent faire [...] Par contre, nous, on a fait très attention que l'association contre l'aéroport de Y n'ait pas de teinture politique. » (Entretien $n^{\circ} 9$ ).

Une deuxième originalité régionale a été la forte implication de professionnels de l'aéronautique, travaillant à Toulouse et résidant dans les campagnes voisines du nord de l'agglomération, à proximité de l'aéroport de Blagnac et du site AéroConstellation. Venus pour la plupart s'installer récemment dans la région et symboles de son dynamisme démographique, ces actifs se sont rapidement impliqués et ont occupé une place centrale :

"L'association de X a été une des premières, parce que les premiers sensibilisés ont été les professionnels de l'aviation qui y habitent. C'est ceux qui connaissaient bien le problème » (Entretien $n^{\circ} 6$ ) ;

"Les cadres d'Airbus se sont les plus féroces contre l'aéroport 》 (Entretien n¹3).

Ingénieurs, cadres, hauts responsables ont apporté des compétences indéniables à la mobilisation, en participant notamment à la réalisation de contre-expertises. Ils ont globalement donné au mouvement une crédibilité technique et scientifique dans le domaine aérien. Mais leur implication a également été perçue comme paradoxale par de nombreux locaux :

"On a quand même des commerciaux d'Airbus dans l'association. Ils sont contre et d'ailleurs c'est eux qui sont les plus contre [...] Alors ils construisent, ils vendent des avions, et ils ne veulent pas être embêtés par les avions! 》 (Entretien $\left.n^{\circ} 2\right)$.

Le profil original de ces cadres de l'aéronautique a en effet cristallisé bon nombre de débats. D’une part, certains considéraient leur présence comme un gage de crédibilité pour la mobilisation, tandis que beaucoup d'habitants ne comprenaient pas leur double positionnement. L'opposition au projet d'aéroport se justifiait à leurs yeux, avant tout par l'inutilité de celui-ci. Plusieurs ont ainsi mis au service du mouvement une approche quasi-professionnelle de déconstruction des études officielles, notamment des perspectives de trafic, pour le démontrer.

$\mathrm{Au}$ même titre que la mise en évidence de la figure d'un peintre, leader local de mobilisations contre le TGV Méditerranée, et symbolisant le défenseur par excellence du paysage provençal (Lolive, 2003), ces cadres et ingénieurs de l'aéronautique ont constitué ici un archétype du nouveau résident des campagnes toulousaines, qui révèle la pleine inscription de cette mobilisation dans son contexte régional, économique et démographique. 


\section{«L choix plus fort que l'acquis »: nouveaux/anciens, quelles places dans la mobilisation?}

Ce mouvement se caractérise aussi par une relative surreprésentation des nouveaux arrivants, des dix-quinze dernières années, dont des personnes de retour dans leur région d'origine au moment de la retraite. Venir vivre dans les campagnes toulousaines, de manière choisie, pensée et préparée, semble en effet inciter à une plus forte implication. Cette situation transparait d'ailleurs, souvent avec excès, dans le discours de nombreux interlocuteurs locaux :

\section{«La mobilisation contre l'aéroport, c'est que des gens de l'extérieur, des gens qui sont arrivés 》 (Entretien n8)}

La volonté des nouveaux arrivants de changer de résidence, de « vivre à la campagne », les efforts financiers et les sacrifices consentis pour y parvenir, ont été autant d'incitations à la mobilisation. Particulièrement réactifs et virulents, ils s'opposent à une opération d'aménagement qui remet en cause leurs choix et leur environnement de vie, qui dévalorise leur patrimoine immobilier et qui porte atteinte à leurs nouvelles vies sociale et professionnelle (construction ou rénovation de maisons, création de commerces).

L'aéroport représente pour eux un des maux de la vie urbaine, qu'ils ont précisément voulu quitter. Certains ont d'ailleurs déjà fait l'expérience de vivre à proximité et des nuisances induites :

"Ils arrivent ici pour être bien. Ils partent de grandes villes où pour la plupart ils babitaient près d'un aéroport. Ils trouvent un village sympathique, une maison qui leur plait, et puis le projet arrive!" (Entretien $\left.n^{\circ} 8\right)$;

"Cette nouvelle population est venue pour échapper aux contraintes de la ville et à ces nuisances [...] Ils sont venus chercher une certaine sérénité et une tranquillité, et quand ils ont eu vent de ce projet, ils ont dit: "Qu'est-ce qui nous arrive? Il faut se bouger 》 (Entretien n ${ }^{\circ}$ ).

Plus globalement, leurs origines et leurs trajectoires de vie citadines, associées pour certains à des compétences militantes et professionnelles, leur ont octroyé une place de choix dans la mobilisation (responsabilités, élaboration d'argumentaires, organisation d'actions). Ils ont notamment été influents sur son déroulement, son institutionnalisation et la mise en place d'une organisation pluriscalaire (des associations locales associées à des collectifs régionaux).

Inversement, les implications de beaucoup de natifs ont été moindres et plus tardives :

"Les gens d'ici, ils s'engagent moins, il y a peu d'agriculteurs qui s'impliquent [...] Les habitants de souche, ils sont solidaires mais ils ne sont pas actifs dans l'association 》(Entretien $\left.n^{\circ} 6\right)$.

Malgré certains combats dans la sphère agricole, leurs attitudes illustrent assurément une culture de la protestation moins prononcée, et des difficultés relatives à se rassembler quand la situation l'exigerait (Cavaillé, 1999).

Ces degrés locaux d'implication rendent également compte de tensions avérées, dans certaines communes, entre les pouvoirs municipaux et les associations dites citoyennes. Ces clivages révèlent souvent une conception culturelle, profondément divergente, des hiérarchies au sein de la mobilisation locale : les natifs et les plus anciennement installés, considérant le maire comme la figure naturelle pour porter leur mécontentement, alors que les nouveaux arrivants, exprimant des réticences à son égard, plébiscitent plutôt les structures associatives. 
Les différents indicateurs utilisés ici mettent en évidence des proximités importantes, sociologiques, générationnelles, professionnelles, entre les opposants. La mobilisation contre l'aéroport révèle également des profils et des trajectoires individuelles très variés. Le refus du projet a par exemple été largement partagé. Pour autant, les positionnements ont été diversifiés, entre leaders du mouvement, actifs intermédiaires et populations plus à la marge, entre l'énonciation de visions du monde et de préoccupations locales plus pragmatiques. Certains ont mis en avant la défense de leur univers de vie, celui de leurs enfants et de leurs petits-enfants, alors que d'autres y voyaient des enjeux plus globaux. De nombreux champs et échelles de réflexion se sont ainsi exprimés parmi les acteurs de la mobilisation. Ils correspondent souvent à des profils sociaux, d'âges et d'origines particuliers, qui symbolisent bien les recompositions démographiques en cours en périphérie lointaine de l'agglomération toulousaine.

\section{UN EVENEMENT MARQUANT POUR LES CAMPAGNES TOULOUSAINES ?}

Dans un deuxième temps, considérant qu'« après un conflit, ce n'est pas la même chose qu'avant", nous analyserons la portée innovante de la mobilisation contre l'aéroport, dans sa capacité à rassembler les populations locales, au moment de la contestation et au-delà (Melé, 2003). Notre principale hypothèse de travail était de considérer ce mouvement comme potentiellement fédérateur d'individus et de groupes très hétérogènes, afin de combattre un même projet qui remettait en cause des démarches et des choix de vie partagés. Par sa portée et son importance, cet évènement est apparu assez singulier dans la vie quotidienne de ces campagnes sous influence urbaine grandissante. L'analyse du profil des opposants a révélé l'impact des transformations démographiques en cours sur son déroulement et la nature de la mobilisation. Dans quelle mesure a-t-elle également fait émerger de nouvelles formes de sociabilités locales, au sein de campagnes par ailleurs traversées par des tensions importantes (Lecourt et al., 2004)?

\section{Une mobilisation porteuse de nouvelles proximités locales et régionales}

L'opposition au projet d'aéroport a été à l'origine, de manière assez classique, de nombreux moments singuliers d'échange et de rapprochements. Durant des mois, cette mobilisation a été vectrice d'une forte animation locale. Les manifestations, les réunions publiques, les différentes actions ont été autant d'opportunités de rencontres et de retrouvailles, entre amis d'enfance, entre anciens élèves et professeurs, entre voisins. Cet évènement a ainsi initié des solidarités nouvelles à travers l'opposition collective à un même projet :

"Des gens qui ne se connaissaient pas se sont parlés. Ça a été un mouvement très convivial et j'ai rencontré des gens que je n'aurais jamais connu par ailleurs [...] Maintenant, quand on se croise, on se dit bonjour, on se connait, on discute, on blague, on s'est fait des copains 》 (Entretien $n^{\circ} 4$ ).

Au sein des villages, certains voisins que les années de vie à proximité, les emplois du temps, ou tout simplement, les circonstances n'avaient pas amené à se rencontrer, se sont découverts à travers cette mobilisation, en s'investissant dans les associations, en se rendant aux manifestations ou dans les réunions publiques locales :

"C'est quand même étonnant, nous étions là depuis 10 ans et ça nous a fait connaître dans un rayon de 100 mètres autour de notre domicile des gens que l'on n'avait jamais vu [...] Ça a permis de tisser des liens qui n'auraient peut être jamais existé » (Entretien n9). 
Malgré une relative surreprésentation des classes moyennes et supérieures, cette mobilisation a également permis, occasionnellement, de rassembler lors des principales actions et autour d'un même objectif, des populations de statuts socioéconomiques hétérogènes. L’analyse précédente des profils d'opposants a bien révélé néanmoins, parmi les plus actifs, une relative homogénéité sociale :

"En termes sociologique et culturel, ça a réuni beaucoup d'extrêmes. Ça a conduit à des rencontres étonnantes. Si je caricature, c'était comme si dans une manifestation un grand patron et ses délégués syndicaux étaient sous la même banderole [...] C'est une originalité de ce mouvement parce que les autres associations ont un même thème et un même profil de personnes 》 (Entretien $n^{\circ} 9$ ).

De manière plus originale, cette mobilisation a permis des rapprochements entre des populations hétérogènes par leurs origines et leurs âges. Les associations locales contre l'aéroport, en premier lieu le cercle des plus impliqués, ont été l'occasion de partages et de combats en commun entre de jeunes couples, nouvellement installées, et des populations plus âgés, retraités, natives pour une bonne part ou d'implantations anciennes. Cette mobilisation a également initié des proximités entre de jeunes locaux, attachés et investis dans la défense de leur territoire de vie, et des nouveaux arrivants de tous âges, qui se sont retrouvés autour d'une même vision des sociétés locales et d'un besoin de les (re)dynamiser. Ces convergences se sont, par exemple, perpétuées au sein d'autres structures associatives, à travers le développement de projets culturels en milieu rural, et au service d'une animation locale enrichie et diversifiée. Les circonstances de vie contemporaines au sein des campagnes périurbaines offrent peu de moments propices à de telles rencontres ; la mobilisation contre l'aéroport a indéniablement été l'une d'elles.

À l'échelle régionale, ce mouvement de contestation a créé des formes de solidarités, originales et transversales, entre populations résidentes de l'ensemble des campagnes toulousaines. Des coopérations associatives et politiques se sont développées entre des communes, de cantons et de départements différents. Ce fut, par exemple, l'occasion pour les élus les plus impliqués, de rencontres et de débats au sein des collectifs régionaux contre le projet d'aéroport, mais aussi d'échanges sur les difficultés de la vie politique locale, dans un contexte commun de recompositions démographiques de leurs campagnes sous l'influence de Toulouse. Au même titre que la mise en place récente de structures intercommunales (communauté de communes, pays), la mobilisation contre l'aéroport a permis de partager des expériences, de dépasser certaines rivalités et « guerres de clochers » ancestrales, et de fait, d'initier de nouvelles proximités spatiales :

"On a discuté d'autres choses, comment fonctionnent nos villages, le développement économique, les schémas de cohérence territoriale, les communautés de communes... Ça a permis des échanges nouveaux, intéressants et concrets, et on ne pourra pas s'oublier [...] 》 (Entretien $\left.n^{\circ} 13\right)$.

Cette mobilisation a été une occasion singulière de rassemblement des populations. Elle a fait naître certaines relations sociales et spatiales à travers un mouvement revendicatif, à la fois local et régional. Sa convivialité est aussi à souligner ; beaucoup de participants étaient d'ailleurs quelque peu nostalgiques de ces mois d'opposition :

"On est tous là à se demander quand est-ce que ça repart, avec une certaine envie, pour l'ambiance, l'émulation et l'aspect festif de ce mouvement 》 (Entretien nº 8 ).

Si l'originalité et les apports de ce mouvement sont indéniables, ses capacités fédératrices sont tout de même restées limitées. Une forte cohésion s'est développée parmi les plus actifs. De nouveaux arrivants ont pu y trouver une manière de s'intégrer aux sociétés locales, mais seuls de petits groupes et espaces ont été véritablement concernés. Les situations ont également varié entre communes, en fonction des relations plus ou moins bonnes existant au sein des structures 
associatives et municipales. Les plus petits villages sont notamment apparus les plus fédérateurs, à l'inverse des principales communes rurales où les coopérations ont été plus difficiles.

\section{Persistance de nombreuses tensions et fractures locales}

Derrière une tranquillité véhiculée par l'imaginaire collectif, nombreuses sont les campagnes aujourd'hui traversées par d'importantes tensions (Urbain, 2008). A la périphérie de Toulouse, elles sont effectivement prégnantes et persistantes (Brevard, 2005), et la mobilisation contre l'aéroport s'est progressivement révélée un prisme d'observation pertinent de ces conflictualités.

Des rivalités anciennes, des fractures relationnelles et politiques, ont été autant d'obstacles à un véritable rassemblement collectif. Le cas de Verdun-sur-Garonne est de ce point de vue intéressant. Dans cette commune qui regroupe un bourg central et des habitations dispersées, la mobilisation contre l'aéroport a mis en évidence de forts clivages spatiaux à l'échelle locale, entre centre et périphérie. Les leaders associatifs résidaient principalement en périphérie du cœur villageois, dans un hameau situé sur un plateau excentré, qui a connu l'arrivée récente de nouvelles populations venues s'installer dans d'anciennes maisons et fermes :

"La mobilisation contre l'aéroport, c'est beaucoup les gens de X, à 4-5 km du village. Beaucoup des gens de l'association vivent là-bas, à proximité » (Entretien $\left.n^{\circ} 1\right)$.

Une segmentation effective et symbolique s'est progressivement instaurée au fil des mois de mobilisation, qui est de fait entrée en résonance avec des dissensions spatiales historiques. Ainsi, l'organisation de la mobilisation locale contre l'aéroport a reproduit des clivages territoriaux préexistants, entre deux entités locales (cœur villageois et hameau périphérique), qui ont été un frein indéniable à la diffusion et à l'intensité communale du mouvement.

Des oppositions plus récentes, révélatrices des transformations démographique et sociale des campagnes toulousaines, sont aussi apparues. Certaines tensions entre municipalités et associations ont ainsi révélé des divergences de profils d'acteurs individuels. Les actifs des associations, plutôt jeunes, nouvellement arrivés et fortement féminisés, contrastaient avec des élus surtout masculins, symboles d'une organisation de notables, plus âgés et originaires de la région.

Cette mobilisation a aussi contribué à l'émergence de personnalités dans la vie publique locale, par l'intermédiaire de la sphère associative, mais leurs profils n'ont fait que renforcer les segmentations énoncées. Ils ont été perçus comme une remise en cause des hiérarchies symboliques locales, ce qui révèle encore une fois des différences de perception des places des acteurs dans le mouvement.

De plus, si ces nouveaux représentants locaux issus de la mobilisation contre l'aéroport ont acquis une large visibilité publique, ils n'ont pas pour l'instant véritablement participé - au sein de nos terrains d'étude - à une remise en cause des structures politiques locales, par exemple au cours des dernières élections municipales de 2008.

Ce mouvement a enfin conduit à une transformation du paysage associatif local. Même si des réseaux préexistants ont pu contribuer au développement de la mobilisation, son expansion a entraîné la création de structures spécifiques. Les associations contre l'aéroport, au même titre que d'autres organisations culturelles et sociales pour lesquelles les implications de nouveaux arrivants sont également fortes, ont participé à un renouvellement du secteur associatif local. Elles sont le signe des transformations démographiques en cours, de nouvelles attentes, de nouveaux centres d'intérêt, et sont indéniablement porteuses de nouvelles ruralités. Les tensions observées durant la mobilisation rendent aussi compte des enjeux importants de pouvoir que représente le secteur associatif local, entre anciennes et nouvelles structurations sociales. 
Il convient tout de même de relativiser les oppositions qu'on vient de relier à l'origine et à l'ancienneté des populations. Les nouveaux arrivants recouvrent en effet une grande variété de profils, correspondant à des choix et des trajectoires de vie hétérogènes. Une même diversité s'exprime parmi les locaux, à travers notamment des comportements générationnels très différents. Cependant, les types de confrontation et de tensions présentés traversent de nombreuses campagnes contemporaines, révélant ici des visions divergentes de la mobilisation, mais surtout un renouvellement en cours des populations, des sociabilités et encore des mentalités locales; ce mouvement n'a au final permis de dépasser ces clivages qu'occasionnellement.

«Le sujet n'est pas d'actualité. L'aéroport de Blagnac fonctionne très bien et la future ligne TGV est faite pour réduire le nombre de passagers aériens"

(J-F Carenco, Préfet de Région, La Dépếche du Midi, 8 Octobre 2007)

Le projet d'un nouvel aéroport dans la région de Toulouse est aujourd'hui abandonné. Le Grenelle de l'environnement a grandement contribué à l'enterrer et à privilégier une liaison ferroviaire à grande vitesse avec Paris, via Bordeaux, prévue à l'horizon 2020. Ainsi, à court et moyen terme, le transport aérien régional continuera de reposer exclusivement sur l'aéroport de Blagnac, situé à proximité directe de l'agglomération. De fait, les gênes subies par les milliers d'habitants voisins, de la périphérie toulousaine proche, leur laissent peu d'espoir d'amélioration dans un avenir proche.

Difficile de dire quelles réelles incidences, sociales et politiques, à plus long terme, cette mobilisation aura eu pour les populations et les vies locales au sein des campagnes toulousaines. Quel sera le devenir des nouvelles proximités, plus ou moins originales, et des sociabilités émergentes observées? Même si ses apports durant le mouvement sont apparus limités et très différents selon les situations locales, l'importance de cette mobilisation et sa singularité ont été indéniables. Nos analyses rendent également compte de la prégnance de fortes tensions locales, qui ont été autant d'obstacles à l'amplification du mouvement. Plus globalement, ces conflictualités, ces clivages, doivent être considérés comme des problématiques centrales des campagnes contemporaines, et en premier lieu, dans la mise en place de politiques de développement rural.

\section{Références bibliographiques}

ARLAUD, S. ; JEAN, Y. ; ROYOUX, D. (sous la dir. de) 2005. Rural-Urbain : Nouveaux liens, nouvelles frontières, Rennes, Presses universitaires de Rennes.

BOSSUET, L. 2007. "Les conflits du quotidien en milieu rural : étude à partir de cinq communes », Géographie, Economie, Société n 2, p. 141-164.

BREVARD, L. 2005. «L'espace périurbain : un espace théâtre de nouvelles pratiques sociales et spatiales et générateur de fractures locales fortes », dans S. Arlaud ; Y. Jean ; D. Royoux (sous la dir. de), Rural-Urbain: nouveaux liens, nouvelles frontières, Rennes, Presses universitaires de Rennes, p. 255264.

CAVAILLE, F. 1999. L'expérience de l'expropriation, Paris, Adef.

DEAR M. 1993. «Comprendre et surmonter le syndrome NIMBY», 2001 Plus n² 27, p. 2-19.

FABUREL, G. 2003a. «Lorsque les territoires locaux entrent dans l'arène publique : retours d'expérience en matière de conflits aéroportuaires », Espaces et Sociétés nº 115, p. 123-146. 
FABUREL, G. 2003b. «Le bruit des avions, facteur de révélation et construction de territoires », L'Espace Géographique n³, p. 205-223.

HALPERN, C. 2007. "Les aéroports entre revendications territoriales et résistances sectorielles » dans A, Faure ; J-P, Leresche ; P, Muller; S, Nahrath (sous la dir. de), Action publique et changements d'échelles : les nouvelles focales du politique, Paris, L'Harmattan, p. 191-203.

JOBERT, A. 1998. "L'aménagement en politique ou ce que le syndrome NIMBY nous dit de l'intérêt général », Politix n 42, p. 67-92.

KAYSER, B. 1990. La renaissance rurale, Paris, Armand Colin.

LAGANIER, J.; VIENNE, D. 2009. «Recensement de la population en 2006 - La croissance retrouvée des espaces ruraux et des grandes villes », INSEE Première $\mathrm{n}^{\circ}$ 1218, $6 \mathrm{p}$.

LECOURT, A. 2003. Les conflits d'aménagement: analyse théorique et pratique à partir du cas breton, Thèse de doctorat, Université de Rennes.

LECOURT, A.; BAUDELLE, G. 2004. «Conflits d'aménagement et proximité sociale: une réévaluation », International Journal of sustainable development $\mathrm{n}^{\circ} 3$, p. 287-301.

LEROUX, M. 2003. "Vers une charte intersonique : Ambiances et espaces sonores », Espaces et Sociétés $\mathrm{n}^{\circ} 115$, p. 105-121.

LOLIVE, J. 1997. «La montée en généralité pour sortir du NIMBY - La mobilisation associative contre le TGV Méditerranée », Politix n³ 39, p. 109-130.

LOLIVE, J. 2003. «Des territoires de mobilisation à l'écorégion : quelques justifications territoriales utilisées par les associations de défense de l'environnement », dans P. Melé ; C. Larrue ; M. Rosemberg (sous la dir. de), Conflits et territoires, Tours, Presses universitaires François Rabelais, p. 145-164.

MARTINEZ, M. 2001. L'impact des nuisances sonores de l'aéroport de Roissy Charles-de-Gaulle sur le marché foncier et l'immobilier, Paris, Rapport ADEF, 30 p.

MELE, P. 2003. «Conflits, territoires et action publique » dans P. Melé ; C. Larrue ; M. Rosemberg (sous la dir. de), Conflits et territoires, Tours, Presses universitaires François-Rabelais, p. 13-32.

PERRIER-CORNET, P. ; HERVIEU, B. 2002. "Les transformations des campagnes françaises : une vue d'ensemble » dans P. Perrier-Cornet (sous la dir. de), Repenser les campagnes, Paris, DATAR, p. 9-33.

PISTRE, P. 2006. La contestation face au projet d'une grande infrastructure de transport: l'exemple du second aéroport toulousain dans le canton rural en recomposition de Verdun-sur-Garonne, Mémoire de Master de Géographie, sous la direction de F. Cavaillé et O. Orain, Université Toulouse-le-Mirail.

SAINT-JULIEN, T. 1985. La diffusion spatiale des innovations, Montpellier, GIP Reclus.

SAUVEE, S. 2004. "La mobilisation riveraine contre le projet de TGV-Ouest, Le NIMBY en Mayenne », Strates n¹1, http://strates.revues.org/index414.html, Consulté le 11 juin 2010.

SIMARD, M. 2007. «Nouvelles populations rurales et conflits au Québec : regards croisés avec la France et le Royaume-Uni », Géographie, Economie, Société n², p. 187-213.

SIMMEL, G. 1995. Le conflit, Paris, Circée.

TOURAINE, A. (sous la dir. de) 1982. Mouvements sociaux d'aujourd'bui, Paris, Ed. Ouvrières.

TORRE, A. 2008. "Activité conflictuelle et processus de gouvernance dans les espaces ruraux français » dans Colloque ARF-LER Ruralités européennes contemporaines : diversité et relations sociales, Lyon, 12-14 Novembre.

URBAIN, J-D. 2008. Paradis verts - Désirs de campagne et passions résidentielles, Paris, Payot. 
Annexe 1 - Entretiens réalisés au sein et à proximité du canton de Verdun-sur-Garonne (82)

\begin{tabular}{|c|c|c|c|c|c|c|}
\hline Entretiens & Age & CSP & $\begin{array}{c}\text { Période } \\
\text { d'installation }\end{array}$ & $\begin{array}{c}\text { Responsabilités } \\
\text { locales }\end{array}$ & $\begin{array}{c}\text { Degrés } \\
\text { d'opposition }^{1}\end{array}$ & $\begin{array}{c}\text { Degrés } \\
\text { d'implication }^{1}\end{array}$ \\
\hline 1. $\mathrm{Mr}$ & 65 ans & Retraité & Natif & & Faible & Faible \\
\hline $\begin{array}{l}\text { 2. } \mathrm{Mr} \\
\text { 2. Mme }\end{array}$ & 45 ans & Agriculteurs & Natifs & Associatives & Fort & Intermédiaire \\
\hline $\begin{array}{l}\text { 3. } \mathrm{Mr} \\
\text { 3. Mme }\end{array}$ & 65 ans & Retraités & Années 1990 & & Intermédiaire & Faible \\
\hline 4. $\mathrm{Mr}$ & 50 ans & $\begin{array}{c}\text { Profession } \\
\text { intellect/sup }\end{array}$ & Années 1980 & & Fort & Fort \\
\hline 5. $\mathrm{Mr}$ & 30 ans & Cadre & Non résident & $\begin{array}{l}\text { Communauté de } \\
\text { communes }\end{array}$ & Faible & Faible \\
\hline 6. $\mathrm{Mr}$ & 60 ans & Retraité & Années 1990 & & Fort & Fort \\
\hline 7. Mme & 60 ans & Retraité & Années 2000 & & Intermédiaire & Faible \\
\hline $\begin{array}{l}\text { 8. } \mathrm{Mr} \\
\text { 8. Mme }\end{array}$ & 45 ans & Employés & Années 1980 & & Fort & Fort \\
\hline 9. $\mathrm{Mr}$ & 65 ans & Retraité & $\begin{array}{c}\text { Mme native, } \\
\text { Mr Années } 1990\end{array}$ & & Fort & Très fort \\
\hline 10. $\mathrm{Mr}$ & 40 ans & Employé & Natif & Associative & Intermédiaire & Intermédiaire \\
\hline 11. Mme & 65 ans & Retraité & Années 1960 & Municipalité & Intermédiaire & Intermédiaire \\
\hline 12. Mme & 35 ans & $\begin{array}{c}\text { Chef } \\
\text { d'entreprise }\end{array}$ & Années 2000 & & Faible & Faible \\
\hline 13. Mme & 55 ans & Inconnue & $\begin{array}{c}\text { Native, de retour } \\
\text { Années } 1990\end{array}$ & Municipalité & Fort & Très fort \\
\hline 14. $\mathrm{Mr}$ & 80 ans & Retraité & Natif & Associatives & Intermédiaire & Faible \\
\hline $\begin{array}{l}\text { 15. Mr } \\
\text { 15. Mme }\end{array}$ & 60 ans & Retraités & $\begin{array}{c}\text { Mme native, } \\
\text { Mr Années } 1970\end{array}$ & & Fort & Très fort \\
\hline 16. $\mathrm{Mr}$ & 40 ans & Ingénieur & Années 1990 & & Fort & Très fort \\
\hline 17. Mme & 35 ans & Employé & Natif & Associative & Fort & Fort \\
\hline 18. $\mathrm{Mr}$ & 50 ans & $\begin{array}{c}\text { Profession } \\
\text { intellect/sup }\end{array}$ & Non résident & & Fort & Très fort \\
\hline
\end{tabular}

${ }^{1}$ Les intitulés attribués à chaque interlocuteur correspondent à une interprétation par l'auteur de la teneur des discours recueillis lors des entretiens semi-directifs :

- 3 modalités ont été retenues pour qualifier l'avis sur le projet d'aéroport : Faible (indifférent, fataliste, mitigé), Intermédiaire (contre le projet, opposition de principe), Fort (virulent, réactif, argumenté)

- 4 modalités ont été retenues pour qualifier le degré d’implication dans le mouvement : Faible (aucune, soutien moral), Intermédiaire (membre d'une association, pétition, participation à des réunions/ manifestations), Fort (membre actif d'une association, participation/organisation des actions), Très fort (responsable et/ou fondateur d'une association communale, représentant au sein des collectifs régionaux, leaders). 